\title{
BMJ Open Protocol for a prospective, observational cohort study of awareness in mechanically ventilated patients admitted from the emergency department: the ED-AWARENESS study
}

\author{
Ryan D Pappal, ${ }^{1}$ Brian W Roberts (1) , ${ }^{2}$ Nicholas M Mohr, ${ }^{3}$ Enyo Ablordeppey, ${ }^{4}$ \\ Brian T Wessman, ${ }^{4}$ Anne M Drewry, ${ }^{5}$ Yan Yan, ${ }^{6,7}$ Marin H Kollef, ${ }^{8}$ \\ Michael Simon Avidan, ${ }^{5}$ Brian M Fuller ${ }^{4}$
}

To cite: Pappal RD, Roberts BW, Mohr NM, et al. Protocol for a prospective, observational cohort study of awareness in mechanically ventilated patients admitted from the emergency department: the EDAWARENESS study. BMJ Open 2019;9:e033379. doi:10.1136/ bmjopen-2019-033379

- Prepublication history and additional material for this paper are available online. To view these files, please visit the journal online (http://dx.doi. org/10.1136/bmjopen-2019033379).

Received 02 August 2019 Revised 12 September 2019 Accepted 16 September 2019

Check for updates

(C) Author(s) (or their employer(s)) 2019. Re-use permitted under CC BY-NC. No commercial re-use. See rights and permissions. Published by BMJ.

For numbered affiliations see end of article.

Correspondence to

Dr Brian M Fuller;

fullerb@wusm.wustl.edu

\section{ABSTRACT}

Introduction Awareness with paralysis is a complication with potentially devastating psychological consequences for mechanically ventilated patients. While rigorous investigation into awareness has occurred for operating room patients, little attention has been paid outside of this domain. Mechanically ventilated patients in the emergency department (ED) have been historically managed in a way that predisposes them to awareness events: high incidence of neuromuscular blockade use, underdosing of analgesia and sedation, delayed administration of analgesia and sedation after intubation, and a lack of monitoring of sedation targets and depth. These practice patterns are discordant to recommendations for reducing the incidence of awareness, suggesting there is significant rationale to examine awareness in the ED population.

Methods and analysis This is a single centre, prospective cohort study examining the incidence of awareness in mechanically ventilated ED patients. A cohort of 383 mechanically ventilated ED patients will be included. The primary outcome is awareness with paralysis. Qualitative reports of all awareness events will be provided. Recognising the potential problem with conventional multivariable analysis arising from a small number of events (expected less than 10-phenomenon of separation), Firth penalised method, exact logistic regression model or penalised maximum likelihood estimation shrinkage (Ridge, LASSO) will be used to assess for predictors of awareness.

Ethics and dissemination Approval of the study by the Human Research Protection Office has been obtained. This work will be disseminated by publication of peer-reviewed manuscripts, presentation in abstract form at scientific meetings and data sharing with other investigators through academically established means.

\section{INTRODUCTION}

\section{Background and rationale}

Awareness is the explicit recall of sensory perceptions during anaesthesia and can carry catastrophic psychological sequelae, as up to

\section{Strengths and limitations of this study}

This study is the largest and most rigorous examination of awareness with paralysis outside of the operating room.

- The observational design will allow the enrolment of a large sample of diverse patients, which will add significantly to the knowledge base regarding awareness in mechanically ventilated emergency department patients.

- An observational design can only describe associations and not causation.

- The study could induce a Hawthorne-type effect among clinicians if they are cognizant of an ongoing study regarding awareness.

$70 \%$ of patients who have experienced awareness suffer from the horror and intense fear of post-traumatic stress disorder (PTSD). ${ }^{1}$ Given this, the Joint Commission on Accreditation of Healthcare Organizations has recommended rigorous efforts be made to prevent awareness. Different methods have been used to detect awareness events, including spontaneous reporting and the Brice questionnaire, with no compelling evidence of improved accuracy of one method versus another. ${ }^{2-4}$ As measured by the Brice questionnaire, the incidence of awareness during anaesthesia with potent inhaled anaesthetics in the operating room (OR) is approximately 1-2 cases $/ 1000^{1}$; however, in high-risk patients given only intravenous anaesthesia, the incidence of awareness approaches $1 \% .{ }^{15}$ While rigorous investigation into awareness has occurred in the OR, much less data exist for patients outside of that domain. This suggests that mechanically ventilated patients in other clinical locations may be at higher risk for 
awareness and the commensurate side effects associated with it.

There is significant rationale to examine awareness in patients receiving mechanical ventilation in the emergency department (ED), as these patients have been historically managed in a way that predisposes them to experience awareness. OR-based studies demonstrate that some critical risk factors for awareness include: (1) a total intravenous anaesthetic approach (as opposed to inhaled); (2) underdosing of anaesthesia; (3) neuromuscular blocker (NMB) use and (4) a lack of protocolled monitoring of sedation depth. ${ }^{1}$ Critically ill ED patients requiring mechanical ventilation almost exclusively receive intravenous medications, and are frequently underdosed. ${ }^{6-8}$ Further, a significant percentage (up to $45 \%)$ of ED patients have been documented in previous literature to receive no analgesia or sedation after intubation. $^{8}$ Approximately $90 \%$ of patients receive an NMB for intubation in the ED, with a recent trend of increased use of long-acting NMB for intubation (ie, rocuronium vs succinylcholine), and up to $25 \%$ receive a long-acting NMB after intubation. ${ }^{7-10}$ In the ED, these paralysed patients typically receive less analgesia and sedation, lower doses and in a delayed fashion. ${ }^{7}$ These practice patterns are completely discordant to recommendations for reducing the incidence of awareness, and suggest that patients mechanically ventilated in the ED are at higher risk for this complication.

More recent data from our research group also demonstrates some concerning practice patterns that could predispose patients to awareness during mechanical ventilation in the ED. In a single-centre study on postintubation sedation practices in the ED, we demonstrated: (1) $15 \%$ of patients given no analgesia or sedation in the ED after intubation; (2) in $25 \%$ of patients, a delay of $50 \mathrm{~min}$ or more to receive sedation after NMB use and intubation and (3) no clinical sedation depth assessment for $>33 \%$ of patients. ${ }^{9}$ To build on this single centre experience, the multicentre $(n=15)$ ED-SED study was a prospective cohort study conducted to examine practice patterns and clinical outcomes associated with ED-based postintubation sedation across a diverse sample of academic centres in the USA. ${ }^{11}{ }^{12}$ Congruent with our single centre experience, the results demonstrate a high-risk situation for awareness: (1) $10.8 \%$ of patients received no analgesia or sedation after intubation; (2) $\sim 90 \%$ received NMB for intubation and $9 \%$ were exposed to additional long-acting NMB after intubation; (3) $>25 \%$ of patients did not have sedation depth assessed and (4) two patients were given no analgesia or sedation, yet given an additional long-acting NMB after intubation. This confirms that currently, despite ED-based publications regarding sedation after the initiation of mechanical ventilation, existing treatment guidelines regarding postintubation sedation and evidence-based recommendations to prevent awareness, clinicians are not paying attention to the potential for awareness in immediate postintubation care.
Human factors likely play a role in the historical lack of relevance given to awareness in critically ill mechanically ventilated patients, as cardiovascular instability or an obtunded mental state may lead clinicians to believe patients are incapable of awareness recall. This may lead to the underdosing of analgesics and sedatives. ${ }^{8}$ However, the fifth National Audit Project on accidental awareness in the UK and Ireland documented accidental awareness in critically ill and unstable patients, demonstrating that critical illness does not guarantee that memory of events will not occur. ${ }^{3}$ This report also identified two cases of awareness in intubated ED patients, suggesting that awareness is possible from the very beginning of intubation and initiation of mechanical ventilation in the ED. Case reports from the ED also confirm the terrifying psychological sequelae that can result from awareness with paralysis. ${ }^{13}$ Puller et al reported that $\sim 25 \%$ of patients recalled an intolerable level of distress during rapid sequence intubation, and $\sim 45 \%$ recalled some level of awareness during intubation. ${ }^{14}$ Miner et $a l^{15}$ reported that $4 / 26(15.4 \%)$ patients had recall of intubation, three of whom had a Visual Analogue Scale of 100 (complete recall). Kimball et al reported on 5/10 (50\%) patients with recall of intubation and Smith and Bishop reported that $2 / 34(5.9 \%)$ patients recalled emergent intubation, but did not report location of recall (ie, ED, intensive care unit (ICU), prehospital, ward). ${ }^{16}{ }^{17}$ However, this prior research regarding awareness in mechanically ventilated ED patients is limited for several reasons: (1) publications exist in abstract (ie, non peer-reviewed) form ${ }^{14}$; (2) case series and convenience (ie, non-consecutive) sample methodology ${ }^{13-16}$; (3) non-validated questionnaires to assess for awareness (ie, Likert, Visual Analogue Scale, $1-10$ scale $)^{14-16}$; (4) small sample size $e^{13-17}$ and (5) a focus on awareness of the intubation procedure only, as opposed to intubation and the postintubation mechanical ventilation period. Therefore, to what extent awareness in mechanically ventilated ED patients is a problem remains unclear. This represents a critical knowledge gap, putting thousands of patients at risk for awareness annually in the USA alone. Given the magnitude of disability suffered by patients with awareness, examining this further is imperative.

The above data regarding ED sedation, including that from our research group, demonstrate that a balanced approach to postintubation sedation for mechanically ventilated ED patients is currently lacking. Taken together, their results justify the need for studies focusing on awareness with paralysis in the critically ill mechanically ventilated ED population to assess how common this problem might be. The genesis of early sedation practice is in the ED, and our data demonstrate that this could be an ideal domain to improve care. Focusing the study of awareness almost exclusively to OR patients is a weakness in the field. The ED-AWARENESS study was, therefore, designed with the objective of examining the incidence of awareness with paralysis in mechanically ventilated ED patients. We hypothesise that the incidence of awareness 
with paralysis will be approximately $1 \%-2 \%$, as measured by the modified Brice questionnaire.

\section{METHODS AND ANALYSIS}

\section{Study design}

This is a single centre, prospective cohort study. Data collection began 1 June 2019 and patients will be enrolled for approximately 12 months.

\section{Study population}

The target population for this study is mechanically ventilated adult patients in the ED. Inclusion criteria are: (1) mechanical ventilation via an endotracheal tube in the $\mathrm{ED}$, including patients intubated in the ED and prior to arrival (ie, in the prehospital setting) and (2) age $\geq 18$ years. Over $90 \%$ of these patients receive NMB at some point during their care in the ED, yet awareness with paralysis has not been examined in this cohort. Therefore, these patients are the optimal group to assess for awareness and prior data support the premise that they are high risk for this complication. Exclusion criteria are: (1) death before discontinuation of mechanical ventilation; (2) presence of neurological injury with residual deficit that precludes awareness assessment (eg, acute cerebrovascular accident, intracranial haemorrhage, traumatic brain injury, status epilepticus, sudden cardiac arrest or fulminant hepatic failure) and (3) transfer to another hospital from the ED. We exclude patients dying before discontinuation of mechanical ventilation because they cannot be assessed for awareness. Presence of neurological injury with residual deficit is an exclusion as patients with neurological injury can have depressed levels of consciousness and coma that may preclude an awareness assessment. Furthermore, after discontinuation of mechanical ventilation, it may be impossible to accurately complete an awareness questionnaire in these patients. Those with a pre-existing neurological injury, such as remote cerebrovascular accident or history of seizures, will be eligible for the study. Patients with an acute neurological injury but without residual deficit (eg, minor traumatic brain injury with no deficits) will also be eligible for the study. Transfer to another hospital is an exclusion as these patients would not be able to be assessed for awareness by study team members. Patients will be recruited exclusively from the ED at Barnes-Jewish Hospital/Washington University School of Medicine in St. Louis. Based on the demographics of the patient population routinely presenting to our hospital, the resulting study population is expected to be approximately $45 \%$ female, $50 \%$ white, $45 \%$ African-American and 5\% other races. We expect a similar distribution, and will enrol patients without regard to gender or race. We, therefore, expect that the study findings will hold external validity.

\section{Screening and study initiation}

All participants will be screened in the ED using an electronic trigger, which captures mechanically ventilated ED patients by identifying the receipt of an NMB (eg, succinylcholine and rocuronium), mechanical ventilation orders or an endotracheal intubation procedure note. This trigger is sent as an electronic mail to the principal investigator (PI) in an automated fashion, thereby facilitating efficient screening and patient recruitment. After this automated screen, study team members will assess patients for inclusion and exclusion criteria. All patients satisfying inclusion and exclusion criteria will be enrolled in the study. This has been part of our standard operating procedure for identifying consecutive mechanically ventilated patients for over 5 years. ${ }^{18-20}$ As a screening backup, the respiratory therapists in the ED will also send the study PI an electronic mail each time a patient is placed on mechanical ventilation.

\section{Patient and public involvement}

The patients in this study were not involved in the development of the research question or study design, and will not be involved in recruitment or conduct of the study.

\section{Data}

We anticipate collecting the following baseline characteristics: age, gender, race, weight, height, pre-existing comorbid conditions, vital signs at presentation and pertinent laboratory variables. Comorbid conditions will include: dementia, diabetes mellitus, cirrhosis, congestive heart failure, end-stage renal disease, chronic obstructive pulmonary disease, immunosuppression, malignancy, alcohol abuse and history of psychiatric illness (eg, schizophrenia, bipolar, major depression or anxiety).

ED process of care variables will include: ED length of stay, blood product transfusion, antibiotics, central venous and arterial catheter placement, and vasopressor infusion. Data related to mechanical ventilation will include: location of intubation (ie, prehospital or ED), indication for mechanical ventilation, ventilator mode, tidal volume, positive end-expiratory pressure, set respiratory rate, fraction of inspired oxygen, peripheral oxygen saturation, peak airway pressure and inspiratory plateau pressure.

We will record all data (including dosage) regarding sedation in the ED will include: NMB and induction agents administered to facilitate endotracheal intubation. Subsequent medications related to analgesia and sedation in the ED will also be collected, and will include: opiates, benzodiazepines, propofol, ketamine, dexmedetomidine, etomidate, haloperidol, quetiapine and NMB. Sedation depth in the ED will be recorded as part of routine clinical care, using the Richmond Agitation-Sedation Scale (RASS). ${ }^{21}$

Pertinent clinical data during the first 48 hours after admission in the ICU, including sedation depth, medications (including dosage) administered for the management of analgosedation and the presence of delirium (assessed with the Confusion Assessment Method for the ICU (CAM-ICU)), per routine care) will also be included. Table 1 shows a full description of events for this study. 
Table 1 Schedule of events for this prospective cohort study

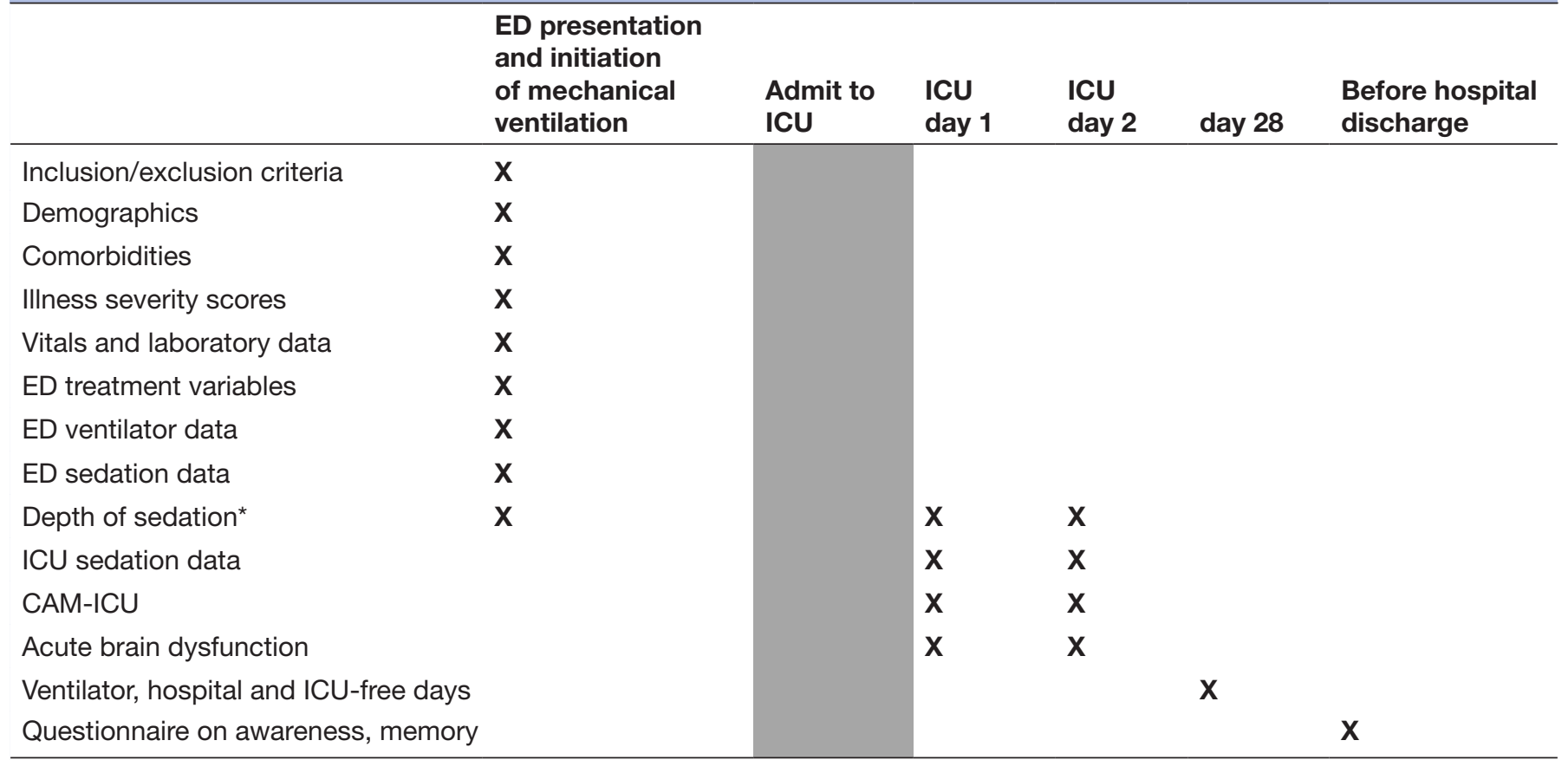

*Assessed with Richmond Agitation-Sedation Scale.

CAM, confusion assessment method; ED, emergency department; ICU, intensive care unit.

\section{Outcomes}

The electronic medical record will be checked daily to see if patients have been extubated. The primary outcome of interest is the incidence of awareness with paralysis. In assessing the outcome in this novel population, it is recognised that the clinical setting and management goals are different between anaesthetised patients in the OR and mechanically ventilated patients in the ED or ICU. In the OR, the goal is to typically achieve deep sedation/ unconsciousness and a lack of movement (ie, paralysis) in the context of intermittent noxious and painful stimulation. In contrast, data from mechanically ventilated critically ill patients demonstrate that achieving light sedation and having an interactive patient will improve outcome. Therefore, the recall of events (ie, memory) from the ICU is fairly common, should be expected and is generally beneficial to the patient. ${ }^{22-25}$ This is in contrast to the limited reports of awareness with paralysis in critically ill mechanically ventilated patients, which have reported on the negative psychological consequences associated with this situation. ${ }^{13}{ }^{26-29}$ Therefore, for the purposes of this study, it is necessary to make a distinction between memory of events and what is typically thought of by the term 'awareness' in the OR: explicit recall of sensory perceptions during intraoperative anaesthesia. To do so, memory and awareness will be assessed with a combination of previously validated questions from the ICU Memory Tool and the modified Brice questionnaire. We will, therefore, purposefully separate memories from awareness with paralysis.
The ICU Memory Tool is a validated questionnaire to assess recall of events from the ICU. ${ }^{22}{ }^{24}{ }^{25}$ Consistent with prior approach, memories will be categorised as: (1) factual; (2) memories of feelings and (3) delusional memories. Regarding awareness with paralysis, we will focus on the time period between losing consciousness and waking up. The Brice questionnaire will assess for awareness with paralysis, and will be modified with targeted supplementary questions in order to assess the specific situation of mechanically ventilated patients in the ED or ICU.

Using this combination of the modified Brice questionnaire and the ICU Memory Tool (online supplementary file 1), we will categorise patients in the following way: (1) memory of events (factual memories, memories of feelings, delusional memories); (2) no memory of events and (3) awareness. Awareness will be present when a patient reports memories of the period between 'losing consciousness' and 'waking up' (questionnaire item \#3 answered as 'yes'). Awareness with paralysis, the primary outcome, will be present when question $8 \mathrm{f}$ of the follow-up questionnaire is answered 'yes', and the patient had documented NMB use. We believe this situation to be most similar to the 'classic' definition of OR awareness with recall. If a patient does not report memories of the period between losing conscious and waking up, but does report memories of wakeful paralysis (ie, recall of endotracheal intubation), then awareness with paralysis will also be considered present. For all patients who report memories of awareness events, we will provide a qualitative report of the patient's subjective reported experience. 
These qualitative reports will essentially be the patients' stories regarding the experience, and will be collected routinely as part of the questionnaire administered to all patients by study team members. Formal analytics will not be applied to these reports; they will be provided to assist with the adjudication of awareness events, and to provide the reader a more transparent description of what the patients' experience were.

Awareness with paralysis will be assessed before hospital discharge, after extubation, provided that no delirium is present at the time of inquiry. After all patients have completed the study, three experts will independently review the responses to the questionnaire from patients who report awareness and determine whether the reported event involved definite awareness with paralysis, possible awareness with paralysis or no awareness with paralysis. Adjudication of awareness will be determined when at least two of the experts are in agreement. For each patient with possible or definite awareness with paralysis, an investigator will use the subjective accounts given by the patients and information in the medical record (ie, clinical setting where procedures occurred or NMB was given, details of intubation) to identify a location (ED vs ICU) and a time window during which awareness with paralysis could have occurred.

Secondary outcomes include perceived threat, acute brain dysfunction, ventilator-free days, ICU-free and hospital-free days. Perceived threat (ie, patient perception of life threat and personal vulnerability) is common among critically ill patients and predisposes them to adverse psychological sequelae such as PTSD. ${ }^{30-32}$ Furthermore, respiratory distress in the ED has been recently shown to be associated with a high degree of perceived threat. ${ }^{33}$ Therefore, we will document perceived threat with a validated threat perception measurement tool. ${ }^{34}$ We will explore links between perceived threat and ED events, including awareness with paralysis. Acute brain dysfunction is a composite outcome composed of delirium and coma. Delirium will be assessed by the CAM-ICU per local institutional protocols. Coma will be defined as having all documented RASS scores of -4 (responsive to only physical stimulus) or -5 (unresponsive) during the first 48 hours. We elect to use this composite outcome since both delirium and coma are major categories of cognitive dysfunction.

\section{Proposed statistical methods}

Descriptive statistics, including mean $\pm \mathrm{SD}$, median (IQR) and frequency distributions, will be used to assess the characteristics of the patient cohort. The incidence of awareness will be described with frequency (\%). To assess predictors of awareness, continuous and categorical variables will be compared using unpaired t-test, Mann-Whitney U test, $\mathrm{X}^{2}$ test or Fisher's exact test, as appropriate. Recognising the potential problem with conventional multivariable analysis arising from a small number of events (expected less than 10-phenomenon of separation), Firth penalised method, exact logistic regression model or penalised maximum likelihood estimation shrinkage (Ridge, LASSO) may be used. If the number of events is very few, we will only perform descriptive analyses. To assess clinical outcomes based on awareness status, $\mathrm{X}^{2}$ (Fisher's exact test) and Kruskal-Wallis tests will be used to compare these outcomes between two groups. All tests will be two tailed, and a $\mathrm{p}<0.05$ will be considered statistically significant.

\section{Sample size}

Patients will be enrolled for approximately 12 months in order to: (1) decrease the chance that any seasonal/ temporal trends could skew the data (eg, higher percentage of patients with trauma in the summer, influenza in the winter) and (2) achieve an adequate sample size. As this is an observational study, the primary outcome of awareness with paralysis is more descriptive than inferential on a hypothesis test between two treatment groups. The required sample size should, therefore, be large enough to observe an event with a high degree of probability and with sufficient precision. Over the course of a year, we expect 2.1 patients per day to fulfil inclusion criteria and be screened for the study, based on our prior work in mechanically ventilated ED patients. ${ }^{12}{ }^{18}$ Based on our prior experience, we expect the following exclusions: (1) death before extubation, $\sim 15 \%$; (2) neurological injury, $30 \%$; (3) transfer to another hospital, $<1 \%$; (4) attrition/refusal to answer the questionnaire, $~ 5 \%$. With an inclusion of just over one patient per day, on average, we expect to enrol 383 patients in the study during the year.

Table 2 demonstrates the incidence of awareness in major randomised controlled trials from the OR, as well as observational studies from the ICU and ED. In the study by Zhang et al, all patients received total intravenous anaesthesia (ie, not inhaled), which is the same route of sedation administration for patients sedated in the ED; the incidence of awareness in the routine care group approached $1 \% .^{5}$ Similarly, all patients in the current proposal will experience routine care. Despite the fact that $>800000$ patients are mechanically ventilated annually in the ICU in the USA, data regarding awareness with paralysis from the ICU population is sparse, and data from the ED is even more sparse. However, there is a concerning discrepancy between the low incidence of awareness in OR patients and the reported rates in the ICU and ED. Given the methodological limitations that exist in the data from the ICU and ED, with high potential for event rate inflation, we choose to make conservative estimations as to the event rate for the population in this study.

Table 3 demonstrates a sensitivity analysis for a range of incidence rates for awareness with paralysis. Since data demonstrate that our patient population is higher risk for awareness when compared with patients managed with total intravenous anaesthesia in the OR, we estimate an incidence of $1 \%-2 \%$. Within this range, the lower bound of the $95 \%$ CI is similar to or higher than the incidence 
Table 2 Sample size justification table

\begin{tabular}{|c|c|c|c|}
\hline Study & Study location & Design & Incidence of awareness, \% \\
\hline Myles et al, $2004^{36}$ & OR & Multicentre RCT & $2.0^{*}$ \\
\hline Avidan et al, $2008^{37}$ & OR & Multicentre RCT & $0.46^{*}$ \\
\hline Avidan et al, $2011^{38}$ & OR & Multicentre RCT & $0.49^{*}$ \\
\hline Zhang et al, $2011^{5}$ & OR & Multicentre RCT & $0.55^{*}$ \\
\hline Mashour et al, $2012^{39}$ & OR & Single centre RCT & $0.19^{*}$ \\
\hline Wagner et al, $1998^{29}$ & $\operatorname{ICU}(n=11)$ & Prospective observational & 36.4 \\
\hline Kaplan et al, $2000^{28}$ & $\operatorname{ICU}(n=57)$ & Single centre before-after & 13.5 \\
\hline Smith et al, $1998^{17}$ & $\mathrm{ED}(\mathrm{n}=34)$ & Prospective observational $\dagger$ & 5.9 \\
\hline Miner et al, $2002^{15}$ & $\operatorname{ED}(n=26)$ & $\begin{array}{l}\text { Prospective observational, convenience } \\
\text { sample† }\end{array}$ & 15.4 \\
\hline Kimball et al, $2011^{16}$ & ED $(n=10)$ & Prospective observational† & 50 \\
\hline Puller et al, $2017^{14}$ & $\mathrm{ED}(\mathrm{n}=53)$ & $\begin{array}{l}\text { Prospective observational, convenience } \\
\text { sample† }\end{array}$ & 24.5 \\
\hline
\end{tabular}

*Indicates overall incidence of definite or possible awareness in both arms of the study.

†Assessed recall of intubation only.

ED, emergency department; ICU, intensive care unit;OR, operating room; RCT, randomised controlled trial.

of awareness seen in the OR. We will, therefore, achieve an appropriate level of precision with high probability. In reality, demonstrating only one event would be extremely meaningful, as: (1) the ED-AWARENESS study is the largest and most rigorous evaluation of awareness with paralysis in this cohort to date and (2) this would translate into approximately 1000 annual cases of awareness in mechanically ventilated ED patients. If only one patient experiences awareness with paralysis, the corresponding incidence of $0.26 \%$ is similar to that seen in the OR population where sedation depth is much more rigorously monitored, and much lower than the reported incidence in ED-based and ICU-based studies. Therefore, we believe that the sample size is adequate to investigate the

\begin{tabular}{|c|c|c|}
\hline $\begin{array}{l}\text { No of patients with } \\
\text { awareness }\end{array}$ & Incidence rate, \%* & $\%, 95 \% \mathrm{Cl}$ \\
\hline 1 & 0.26 & 0.01 to 1.4 \\
\hline 2 & 0.52 & 0.1 to 1.9 \\
\hline 3 & 0.78 & 0.2 to 2.3 \\
\hline 4 & 1.0 & 0.3 to 2.7 \\
\hline 5 & 1.3 & 0.4 to 3.0 \\
\hline 6 & 1.6 & 0.6 to 3.4 \\
\hline 7 & 1.8 & 0.7 to 3.7 \\
\hline 8 & 2.1 & 0.9 to 4.1 \\
\hline 9 & 2.3 & 1.1 to 4.4 \\
\hline 10 & 2.6 & 1.3 to 4.7 \\
\hline 11 & 2.9 & 1.4 to 5.1 \\
\hline
\end{tabular}

${ }^{*}$ Based on a total enrolment of 383 patients. objectives of the study and there is a high probability for an event to occur.

\section{Anticipated results}

We anticipate that the incidence of awareness with paralysis will be $1 \%-2 \%$.

\section{Data storage and management}

All data will be entered by the study team and data accuracy will be verified by the study PI. Data quality control measures will include queries to identify missing data, outliers and discrepancies. Only study team members will have access to protected health information. After enrolment, a unique identifier will be assigned to each study subject. The data will be uploaded and stored using Research Electronic Data Capture, a web-based data management application. All computers will be password protected and encrypted per university policy. We will ensure that the anonymity is maintained. Patients will not be identified by name in any reports on this study. The study PI will have access to the final study dataset.

\section{Dissemination and data sharing}

To enhance reporting transparency, this study will be reported in accordance with the Strengthening the Reporting of Observational Studies in Epidemiology Statement: Guidelines for Reporting Observational Studies. ${ }^{35}$

Data and resources will be shared with other eligible investigators through academically established means. The datasets used and/or analysed during the study will be available from the corresponding author on reasonable request. Collaboration with others investigators interested in optimising outcomes for mechanically ventilated patients in the ED will be welcomed. The results 
from this work will be published as a full-length, peer-reviewed manuscript and presented at national meetings.

\section{Strengths and limitations}

Strengths

The ED-AWARENESS study will add a significant amount of data regarding awareness in non-OR patients, and will address some of the prior weaknesses in this field. It will be the largest study to date on the topic of awareness outside of the OR, and will prospectively enrol consecutive patients. The questionnaire is rigorous and will systematically separate memories from potential awareness events, and we will also capture data regarding intubation as well as the postintubation period. The pragmatic enrolment of a large, diverse sample of mechanically ventilated ED patients will increase the external validity of our findings.

\section{Limitations}

This study will have several limitations. Due to the lack of data regarding awareness in this population, it is possible that an event will not be detected. Based on the data regarding awareness in OR patients managed with total intravenous anaesthesia, along with the limited data from the ICU and ED, we believe this to be unlikely. Further, data from our research group suggests ED patients have sedation managed in a way that increases risk for awareness. We, therefore, believe that our sample size and enrolment duration is adequate to achieve the goals of this investigation. A Hawthorne effect could be induced among clinicians if they are aware of the intent of the study. As part of our standard operating procedure, all screening, data collection and questionnaire administration will occur remotely from clinical care provided in the ED. We, therefore, believe it unlikely that we will see a change in clinical behaviour based on the study. As an observational study, we will not necessarily be able to ascribe causation between any awareness events and ED clinical care. This is not the primary intent of this investigation, but rather to record and report the incidence of awareness as rigorously as possible. We have taken rigorous efforts to delineate memory of events from awareness, and to try and differentiate procedural awareness (ie, of the intubation) versus awareness with paralysis while being mechanical ventilated. In reality this may prove quite challenging, requiring us to modify our approach or reporting after the data are collected. In order to improve transparency, we will report all qualitative accounts of awareness events and pertinent clinical events that may provide a window during which awareness could have occurred. By reporting the incidence of awareness with the most comprehensive approach to date, this study represents another step in the process of modifying ED-based postintubation sedation in a way that improves outcomes and reduces adverse events.

\footnotetext{
Author affiliations

${ }^{1}$ Washington University in Saint Louis School of Medicine, Saint Louis, Missouri, USA ${ }^{2}$ Emergency Medicine, Cooper Medical School of Rowan University, Camden, New Jersey, USA
}

${ }^{3}$ Emergency Medicine and Anesthesiology, Roy J. and Lucille A. Carver College of Medicine University of lowa, lowa City, lowa, USA

${ }^{4}$ Anesthesiology and Emergency Medicine, Washington University, Saint Louis, Missouri, USA

${ }^{5}$ Anesthesiology, Washington University in Saint Louis School of Medicine, Saint Louis, Missouri, USA

${ }^{6}$ Clinical Epidemiology Center, VA Saint Louis Health Care System, Saint Louis, Missouri, USA

${ }^{7}$ Public Health Sciences, Washington University in Saint Louis School of Medicine, Saint Louis, Missouri, USA

${ }^{8}$ Medicine, Washington University in Saint Louis School of Medicine, Saint Louis, Missouri, USA

Contributors BMF: conception and study design, acquisition of data, analysis and interpretation of data, drafting and revising the manuscript. RDP: conception and study design, acquisition of data, analysis and interpretation of data, and revising the manuscript. BWR: study design, analysis and interpretation of data, drafting and revising the manuscript. NMM: study design, analysis and interpretation of data, drafting and revising the manuscript. AMD: study design, analysis and interpretation of data, drafting and revising the manuscript. EA: study design, analysis and interpretation of data, drafting and revising the manuscript. BTW: study design, analysis and interpretation of data, drafting and revising the manuscript. YY: study design, analysis and interpretation of data, drafting and revising the manuscript. MHK: study design, analysis and interpretation of data, drafting and revising the manuscript. MSA: study design, analysis and interpretation of data, drafting and revising the manuscript. All authors have read and given final approval of the submitted manuscript.

Funding The authors have not declared a specific grant for this research from any funding agency in the public, commercial or not-for-profit sectors.

\section{Competing interests None declared.}

Patient consent for publication Not required.

Ethics approval The study protocol has received ethical approval by the Human Research Protection Office at Washington University School of Medicine in St. Louis, and will be conducted with waiver of informed consent.

Provenance and peer review Not commissioned; externally peer reviewed.

Open access This is an open access article distributed in accordance with the Creative Commons Attribution Non Commercial (CC BY-NC 4.0) license, which permits others to distribute, remix, adapt, build upon this work non-commercially, and license their derivative works on different terms, provided the original work is properly cited, appropriate credit is given, any changes made indicated, and the use is non-commercial. See: http://creativecommons.org/licenses/by-nc/4.0/.

ORCID iD

Brian W Roberts http://orcid.org/0000-0002-7690-997X

\section{REFERENCES}

1 Avidan MS, Mashour GA. Prevention of intraoperative awareness with explicit recall: making sense of the evidence. Anesthesiology 2013;118:449-56.

2 Brice DD, Hetherington RR, Utting JE. A simple study of awareness and dreaming during anaesthesia. Br J Anaesth 1970;42:535-42.

3 Pandit JJ, Andrade J, Bogod DG, et al. 5Th national audit project (NAP5) on accidental awareness during general anaesthesia: summary of main findings and risk factors. $\mathrm{Br} J$ Anaesth 2014;113:549-59.

4 Cook TM, Pandit JJ. Pitfalls of comparing incidences of awareness from NAP5 and from Brice studies. Br J Anaesth 2015;115:471-2.

5 Zhang $\mathrm{C}$, Xu L, Ma Y-Q, et al. Bispectral index monitoring prevent awareness during total intravenous anesthesia: a prospective, randomized, double-blinded, multi-center controlled trial. Chin Med J 2011;124:3664-9.

6 Bonomo JB, Butler AS, Lindsell CJ, et al. Inadequate provision of postintubation anxiolysis and analgesia in the ED. Am J Emerg Med 2008;26:469-72.

7 Korinek JD, Thomas RM, Goddard LA, et al. Comparison of rocuronium and succinylcholine on postintubation sedative and analgesic dosing in the emergency department. Eur J Emerg Med 2014;21:206-11.

8 Weingart GS, Carlson JN, Callaway CW, et al. Estimates of sedation in patients undergoing endotracheal intubation in US EDS. Am J Emerg Med 2013;31:222-6. 
9 Stephens RJ, Ablordeppey E, Drewry AM, et al. Analgosedation practices and the impact of sedation depth on clinical outcomes among patients requiring mechanical ventilation in the ED: a cohort study. Chest 2017;152:963-71.

10 Brown CA, Bair AE, Pallin DJ, et al. Techniques, success, and adverse events of emergency department adult Intubations. Ann Emerg Med 2015;65:363-70. e1.

11 Fuller BM, Mohr NM, Roberts BW, et al. Protocol for a multicentre, prospective cohort study of practice patterns and clinical outcomes associated with emergency department sedation for mechanically ventilated patients: the ED-SED study. BMJ Open 2018;8:e023423.

12. Fuller BM, Roberts BW, Mohr NM, et al. The ED-SED study: a multicenter, prospective cohort study of practice patterns and clinical outcomes associated with emergency department sedation for mechanically ventilated patients. Critical Care Medicine 2019.

13 Finlay T, Parke T. Awareness in the emergency department: a patient's story. J Intensive Care Soc 2016;17:175-7.

14 Puller J, Juhasz K, Zerkle S, et al. 24 PRIER: patient recall in emergency rapid sequence intubation. Ann Emerg Med 2017;70:S11.

15. Miner JR, Haug E, Friewald S, et al. Evaluation of bispectral EEG analysis and recall in paralyzed intubated patients. Ann Emerg Med 2002;9:405-6.

16 Kimball D, Kincaide RC, Ives C, et al. Rapid sequence intubation from the patient's perspective. West J Emerg Med 2011;12:365-7.

17 Smith B, Bishop R. Incidence of recall of emergency intubation: a preliminary report. Emerg Med 1998;10:223-5.

18 Fuller BM, Ferguson IT, Mohr NM, et al. Lung-Protective Ventilation Initiated in the Emergency Department (LOV-ED): a quasiexperimental, before-after trial. Ann Emerg Med 2017;70:406-18.

19 Fuller BM, Ferguson IT, Mohr NM, et al. A quasi-experimental, before-after trial examining the impact of an emergency department mechanical ventilator protocol on clinical outcomes and lungprotective ventilation in acute respiratory distress syndrome. Crit Care Med 2017;45:645-52.

20 Fuller BM, Mohr NM, Miller CN, et al. Mechanical ventilation and ARDS in the ED: a multicenter, observational, prospective, crosssectional study. Chest 2015;148:365-74.

21 Reade MC, Finfer S. Sedation and delirium in the intensive care unit. New England Journal of Medicine 2014;370:444-54.

22 Jones C, Griffiths RD, Humphris G, et al. Memory, delusions, and the development of acute posttraumatic stress disorder-related symptoms after intensive care. Crit Care Med 2001;29:573-80.

23 Russell S. An exploratory study of patients' perceptions, memories and experiences of an intensive care unit. J Adv Nurs 1999;29:783-91.

24 Samuelson KAM, Lundberg D, Fridlund B. Stressful memories and psychological distress in adult mechanically ventilated intensive care patients - a 2-month follow-up study. Acta Anaesthesiol Scand 2007;51:671-8.
25 Svenningsen $\mathrm{H}$. Associations between sedation, delirium and posttraumatic stress disorder and their impact on quality of life and memories following discharge from an intensive care unit. Dan Med $J$ 2013;60:B4630.

26 Ballard N, Robley L, Barrett D, et al. Patients' recollections of therapeutic paralysis in the intensive care unit. Am J Crit Care 2006;15:86-94.

27 Johnson KL, Cheung RB, Johnson SB, et al. Therapeutic paralysis of critically ill trauma patients: perceptions of patients and their family members. Am J Crit Care 1999;8:490

28 Kaplan LJ, Bailey H. Bispectral index (bis) monitoring of ICU patients on continuous infusion of sedatives and paralytics reduces sedative drug utilization and cost. Critical Care 2000;4(Suppl 1).

29 Wagner BK, Zavotsky KE, Sweeney JB, et al. Patient recall of therapeutic paralysis in a surgical critical care unit. Pharmacotherapy 1998;18:358-63.

30 Wiedemar L, Schmid J-P, Müller J, et al. Prevalence and predictors of posttraumatic stress disorder in patients with acute myocardial infarction. Heart Lung 2008;37:113-21.

31 Davydow DS, Zatzick D, Hough CL, et al. A longitudinal investigation of posttraumatic stress and depressive symptoms over the course of the year following medical-surgical intensive care unit admission. Gen Hosp Psychiatry 2013;35:226-32.

32 Meli L, Kautz M, Julian J, et al. The role of perceived threat during emergency department cardiac evaluation and the age-posttraumatic stress disorder link. J Behav Med 2018;41:357-63.

33 Moss J, Roberts MB, Shea L, et al. Healthcare provider compassion is associated with lower PTSD symptoms among patients with life-threatening medical emergencies: a prospective cohort study. Intensive Care Med 2019;45:815-22.

34 Cornelius T, Agarwal S, Garcia O, et al. Development and validation of a measure to assess patients' threat perceptions in the emergency department. Acad Emerg Med 2018;25:1098-106.

35 von Elm E, Altman DG, Egger M, et al. The strengthening the reporting of observational studies in epidemiology (STROBE) statement: guidelines for reporting observational studies. Ann Intern Med 2007;147:573-7.

36 Myles PS, Leslie K, McNeil J, et al. Bispectral index monitoring to prevent awareness during anaesthesia: the B-Aware randomised controlled trial. The Lancet 2004;363:1757-63.

37 Avidan MS, Zhang L, Burnside BA, et al. Anesthesia awareness and the bispectral index. N Engl J Med 2008;358:1097-108.

38 Avidan MS, Jacobsohn E, Glick D, et al. Prevention of intraoperative awareness in a high-risk surgical population. N Engl J Med 2011;365:591-600.

39 Mashour GA, Shanks A, Tremper KK, et al. Prevention of intraoperative awareness with explicit recall in an unselected surgical population: a randomized comparative effectiveness trial. Anesthesiology 2012;117:717-25. 